\title{
Experimental-based Analysis of the Effect of Channel Errors in the Cluster Formation Phase in Wireless sensor Networks
}

\author{
Edgar Romo Montiel, Mario E. Rivero-Ángeles, Herón Molina Lozano, \\ Rolando Menchaca Méndez \\ Instituto Politécnico Nacional, Centro de Investigacion en Computacion \\ Mexico \\ eromom0900@alumno.ipn.mx, \{erivero, hmolina,rmen\}@ cic.ipn.mx
}

\begin{abstract}
In this paper we present an experimental analysis of the performance of the cluster formation phase of a wireless sensor network. Unlike previous works, this analysis considers the effect of channel errors overran adaptive transmission probability scheme that is used in the cluster formation phase. Particularly, we study the impact of channel errors over the energy consumption and the average cluster formation time. The cluster scheme under study is based on the well-known LEACH protocol, which organizes the nodes of a wireless sensor network into groups in order to distribute the energy consumption of each node. However, in order to reduce processing time and energy consumption, we propose a variant of LEACH protocol where nodes are chosen to be cluster heads or cluster members based only on the order of arrival of nodes (called LEACH MOD), instead of whether or not a node has been a cluster head in the past.
\end{abstract}

Keywords: Wireless sensor network, adaptive transmission probability, LEACH protocol, channel error, energy consumption, cluster formation.

\section{Introduction}

Wireless Sensor Networks (WSNs) are technologies that provide a tool to have control, knowledge and monitoring of different sorts of physical variables of interest. WSNs allow collecting information of the environment depending on the application. Furthermore, WSNs use small nodes that can be installed inside a diversity of spaces, like a wall, a roof, under the pavement, among others. Unlike other wireless networks, WSNs require energy efficient communication among nodes in order to extend the system performance and lifetime. Indeed, in these networks, nodes can be located in places where they cannot be easily reached. As such, once the energy of a node is depleted, its battery cannot be replaced. When a certain number of nodes no longer function, the network loses its functionality. Also, nodes are provided only with a battery of small size and capacity [1]. Specifically, the main problems associated to energy depletion of nodes are: Information losses decrease and holes in the coverage area and broken links among nodes or base station (sink). Building on this, the energy 
consumption is even more critical in such scenarios where the coverage area is particularly wide, in that case, this increases due to the high-energy transmissions of nodes in order to reach the sink node.

To reduce the energy consumption in the system and distribute the energy load among nodes, a clustered-based protocol can be used. In this work, a clustering protocol is proposed, based on the well-known LEACH[2] protocol, called LEACHMOD, which operates as follows: a) Nodes are chosen to be cluster heads or cluster members only based on the order of arrival of the nodes to reduce processing time and energy consumption. b) Nodes transmit in the cluster formation phase with a transmission probability $\tau$, which is adapted in each time slot and varies according to the number of nodes remaining to transmit their control packet. As such, the transmission probability increases in each successful transmission. In each time slot the sink calculates the adequate transmission probability based on the estimation of nodes attempting to transmit, even more, it is considered that a channel is not errorfree and has a probability to obtain false negative or false positive errors described in following sections.

The rest of the paper is organized as follows: Section 2 describes the generalities of LEACH protocol, continued by the Proposed clustering scheme in section 3 where are described each consideration for this proposal. The parameters considered in the system model are given in section 4 and section 5 shows the results obtained by such system model. Finally, a brief conclusion is given in section 6 .

\section{LEACH Protocol}

LEACH is a protocol that organizes nodes inside a wireless sensor network in clusters in order to distribute the energy load of each node. Under this scheme, all nodes organize themselves to take part in a unique cluster as cluster head $(\mathrm{CH})$ or as cluster member (CM) [3]. The cluster head of each cluster (group of nodes) has the function of gathering the sensed data of each node that belongs to the cluster. When the cluster head has gathered all the cluster information, it sends the whole data to the sink.

We can easily see that $\mathrm{CH}$ s consume more energy since they have to be constantly receiving data from the $\mathrm{CMs}$, and perform high-energy transmissions to the sink node once that information of the cluster is gathered. This means that $\mathrm{CHs}$ drain their energy faster. For that reason, LEACH gives and rotates the function of cluster heads among all nodes in the network in order to balance the energy consumption equitably. This rotation occurs periodically in time spaces called rounds and considers nodes that have not been $\mathrm{CHs}$ in previous round to be $\mathrm{CHs}$. The $\mathrm{LEACH}$ protocol considers that each round is composed by two phases: the cluster formation phase and steady state phase [4]; after each round, clusters are broken and the cluster formation starts again selecting new $\mathrm{CHs}$. In the cluster formation phase nodes are selected either as $\mathrm{CHs}$ or $\mathrm{CMs}$ and the steady state phase is where clusters are already established and data transmission is achieved from $\mathrm{CMs}$ to $\mathrm{CH}$ inside each cluster. In the former phase, a random access protocol is used. Specifically, the slotted NP-CSMA (NonPersistent Carrier-Sense Multiple Access) scheme is applied, where nodes transmit in each time slot with probability $\tau$. In the steady state phase, a TDMA based scheme is preferred since the $\mathrm{CH}$ already knows the number of nodes that will report data in the 
cluster. Hence, transmissions can be done in an orderly fashion. Nodes will choose to become cluster heads according to the following equation [2]:

$$
T(n)=\left\{\begin{array}{cc}
\frac{P}{1-P x\left(\operatorname{rmod} \frac{1}{P}\right)} & \text { if } n \in G \\
0 & \text { otherwise }
\end{array}\right.
$$

Where $P$ is the percentage of cluster heads into the network, $r$ is the number of current cluster heads and $G$ is the group of nodes that have not been cluster head in a previous time. In this way the protocol allows the adequate distribution of energy consumption in each node. Fig. 1 shows how the network is organized in clusters, where nodes with a blue circle are the cluster heads.

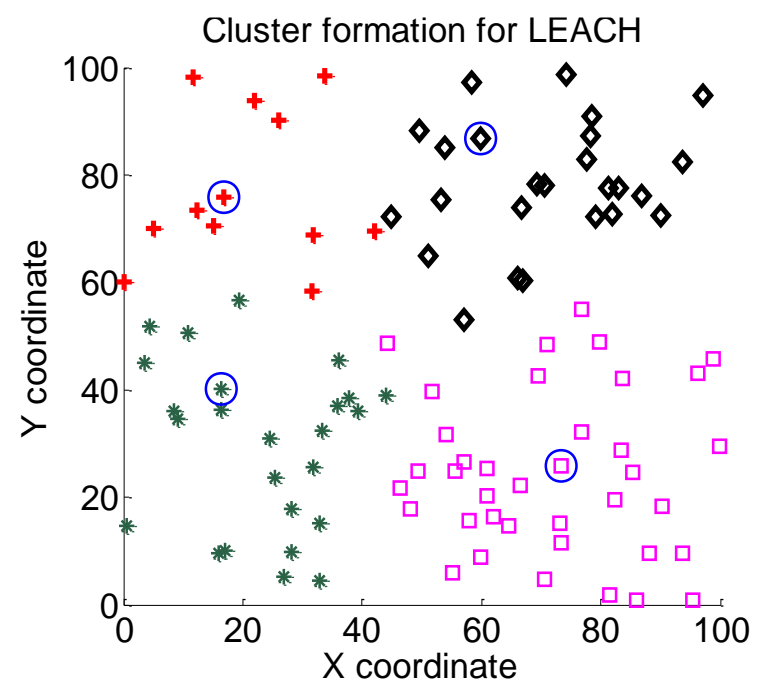

Fig. 1. Network organized in cluster for LEACH [5].

Note that there exist a complete set of protocols for wireless network [6] and especially for WSN [7], [8], [9] and each one of them has certain advantage over the others. Therefore, WSN have flexibility to choice any of those protocols according to the application in order to obtain its best performance [10]. This constantly generates a wide spectrum of research topics.

\section{Proposed Clustering Scheme}

In this work, a clustering protocol based on the well-known LEACH protocol is proposed and studied. This clustering protocol named LEACH-MOD, works as follows. Nodes are chosen to be cluster heads or cluster members only based on the order of arrival of nodes. Specifically, in the cluster formation phase, nodes transmit in each time slot according to a geometric process with probability $\tau$, which is broadcasted by the sink according to its estimation of the outcome of the previous. In 
any given time slot, if there are two or more transmissions, a collision occurs and none of the transmitted packets are received successfully. Consequently the packets involved in the collision have to be retransmitted. On the other hand, if only one packet is transmitted in a time slot, it is considered to be successful and all the nodes in the network recognize such transmission. Building on this, the LEACH-MOD scheme considers that the first $N_{C H}$ nodes that successfully transmit their packet become cluster heads while the rest become cluster members. Fig. 2 shows how the network is organized in clusters based on the LEACH-MOD scheme, where nodes with a blue circle are cluster head.

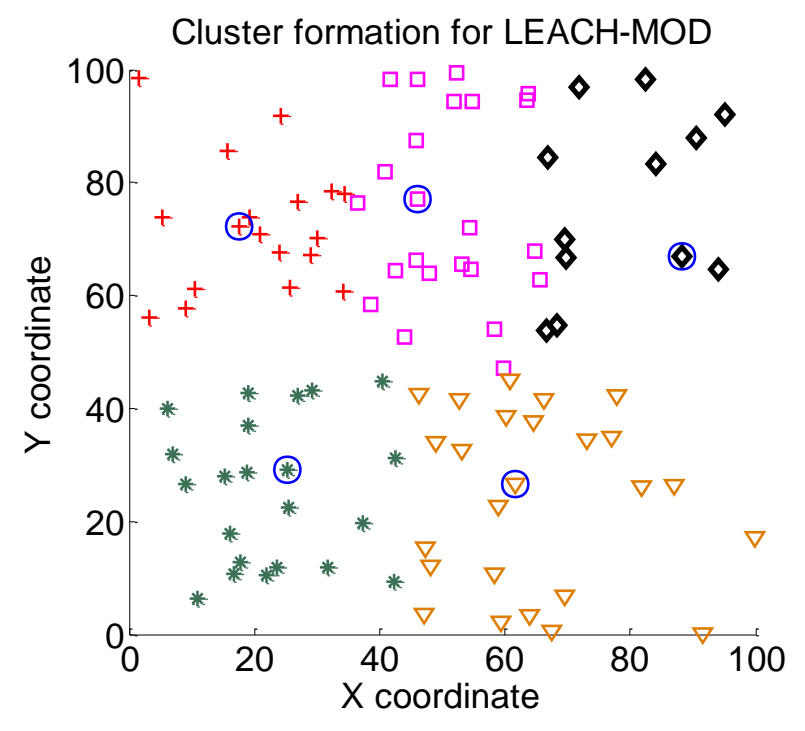

Fig. 2. Network organized in cluster for LEACH-MOD [5].

The main reason to consider this modification to the LEACH protocols is that, in this protocol, each node calculates its transmission probability according to the nodes that have been $\mathrm{CHs}$ before. As such, nodes need to calculate this transmission probability throughout the operation of the network, which entails extra energy consumption and increases complexity. Furthermore, it is possible that each node calculates a different transmission probability. On the other hand, the LEACH-MOD approach does not require this transmission probability computation since the sink computes and broadcast it. It is important to mention that the LEACH protocol ensures that no node can become $\mathrm{CH}$ in successive rounds. This cannot be guaranteed by the proposed protocol. However, since nodes transmit according to a geometric process with parameter $\tau$, the probability that a node is elected as a $\mathrm{CH}$ in consecutive rounds is very low. Also, it can notice that neither LEACH nor LEACH-MOD protocols ensure a uniform distribution of clusters in the system area.

On a more detailed study on the effect of the transmission probability in LEACHMOD, it is possible to see that when the number of nodes with a pending transmission is relatively high, the use of a high value of $\tau$ causes a high amount of collisions inside the cluster formation process, entailing a quick energy drain for all nodes in the 
system due to each node has to retransmit again. On the other hand, the use of a low transmission probability in the same scenario entails a lower number of collisions. However, as the nodes successfully transmit their control packet, the number of pending transmissions decreases accordingly. Hence, the low value of $\tau$ is no longer adequate when only a few nodes remain to transmit their packet since a high idle transmission period occurs. Such that, it is clear that the value of the transmission probability has to vary according to the number of remaining nodes in the cluster formation procedure. As such, we propose an adaptive transmission strategy.

\subsection{Adaptive Transmission}

As mentioned before, for the adaptive transmission probability strategy it was proposed that the value of $\tau$ varies according to the number of nodes with a pending transmission. Hence, the transmission probability of each node depends on the inverse of the number of neighbor nodes for each time slot. In this way, with a high number of contending nodes, the transmission probability is low, and vice versa. From this, each node computes the value of $\tau$ as $\tau_{A}=\frac{1}{i}$. Where $i$ is the number of remaining nodes attempting to transmit in the cluster formation phase. This adaptive transmission probability scheme was studied considering an error-free channel (see [5]), where it is assumed that all nodes know the exact number of nodes attempting a transmission. As it can be seen, in order to calculate the value of $\tau$ the sink estimates the number of nodes with a pending transmission in each time slot of the cluster formation phase for each round, as sink does not have energy restriction issues can perform this estimation and broadcast it to the nodes in the system. This estimation can be done by detecting each successful transmission and assuming that the number of nodes in the system has not changed, i.e., that all initial nodes are still correctly functioning. However the successful transmission detection is not error-free in a wireless environment due mainly to fading, interference and noise.

\subsection{Channel Error}

In order to see how the system is affected by channel errors, we study the effect of error estimation on the performance of the adaptive probability scheme. Specifically, it is considered that two estimation errors can occur in such environment: false positive and false negative estimation. The latter corresponds to the case when the sink node does not detect a transmission while the former corresponds to the case when the sink node detects a transmission but in reality no such transmission occurred. As such, the estimation of $\tau$ can have a higher (in case of false negative estimation) or lower value (in case of false positive estimation) than the actual value $i$. In this work, we assume that either false positive and false negative estimation errors have a probability to occur called PeP and PeN respectively. Different values of these probabilities are considered with the objective of analyzing the effect on the network of a noisy channel. Since the sink calculates the number of remaining nodes in the cluster formation phase according to the outcome of the previous time slot, we differentiate the actual number of nodes, $i$, from the estimation, $i$. Specifically, the channel errors can impact the cluster formation phase as follows: 
Edgar Romo-Montiel, Mario E. Rivero-Ángeles, Herón Molina-Lozano, Rolando Menchaca-Méndez.

1. When there are no transmissions

a. A false positive occurs, then the sink estimates i'-1 nodes attempting to transmit, i.e. the sink assumes that a successful transition occurred.

b. A false negative occurs, then the estimation of the sink, $i \prime$, is the same.

c. It is not possible that both the false positive and false negative errors occur at the same time.

d. Neither false positive nor false negative occur, then there is not a change and the sink estimation, i', remains unchanged.

2. When there is one transmission

a. A false positive occurs, then the sink detects a collision and its estimation $i^{\prime}$ is the same. Note that in this case, the node that transmitted assumes that its transmission suffered a collision (when in fact it did not) and retransmits its packet in a future time slot.

b. A false negative occurs, then the sink does not detect the transmission and its estimation, i', is unchanged.

c. It is not possible that both the false positive and false negative errors occur at the same time.

d. Neither false positive nor false negative errors occur, then there is a successful transmission and the sink estimates $i^{\prime}-1$.In this case, the actual remaining nodes with pending transmission, $i$, decreases in one.

3. When there are two or more transmissions

a. A false positive occurs, then the sink detects a collision and its estimation $i$ ' remains unchanged.

b. We assume that a false negative cannot occur since the energy level in two or more transmission is sufficiently high that it is always detected as a collision. Then, the estimation $i$ ' remains unchanged.

c. It is not possible that both the false positive and false negative errors occur at the same time.

d. Neither false positive nor false negative occur, then there is a collision and the sink estimation $i^{\prime}$ remains unchanged.

From the previous discussion, we consider that the transmission probability in the cluster formation phase is now calculated as $\tau_{A}=\frac{1}{i}$. Note that the transmission probability varies in each time slot according to the estimated outcome of the previous time slot. It is important to note that $i$ always is bigger or equal to $i^{\prime}$. The case where $i$ is equal to $i^{\prime}$, implies that there have not been errors. As more errors occur, $i^{\prime}$ will be smaller than $i$. The critical case is when $i^{\prime}$ is by far smaller than $i$. In this case, there will be high transmission probability for a high number of nodes that attempt a transmission, causing a high collision rate. The lowest value of $i^{\prime}$ is 0 . In this case, $\tau_{A}$ takes the value of 0.95 in order to prevent a catastrophic scenario where nodes transmit in each time slot. 


\section{$4 \quad$ Model of the System}

In this section, the main system parameters and assumptions are presented. It is considered a square coverage area of 100 meters per side with a variable number of nodes in order to analyze the performance of the cluster formation process under different scenarios. The energy consumption model considered is described in detail. We considered normalized energy units as values of energy required for the packet transmission and reception. This is basically to have a general energy consumption model that can be easily scaled to any commercial equipment. Building on this, we assume the following:

- $\quad$ The energy required to transmit a packet in the cluster formation phase $(\mathrm{CF})$ is $E_{t x}^{C F}=0.02$ units.

- The energy required to receive a packet in the cluster formation phase $(\mathrm{CF})$ is $E_{t x}^{C F}=0.01$ units.

- The energy required to transmit a packet from a cluster member to a cluster head in the steady state is $E_{t x}^{\text {Steady }}=0.01$ units.

- $\quad$ The energy consumed by the cluster head in order to receive a packet from a cluster member in the steady state is $E_{t x}^{\text {Steady }}=0.01$ units.

- The energy consumed by the cluster head to transmit a packet to the sink node $(\mathrm{CH} \rightarrow \operatorname{Sink})$, is $E_{t x}^{C H \rightarrow \operatorname{Sink}}=0.2$ units.

Note that the energy required to transmit a packet is always higher than the energy required to receive such packet. Also the packet transmission from the cluster head to the sink node is the most energy consuming. Besides, we consider that the sink has an infinite energy supply. Furthermore, as a novel feature of this work, a non-ideal wireless channel is considered where channel errors can occur. Specifically, all nodes transmit directly to the sink node, and it determines whether a successful transmission, a collision or an empty slot occurs. As such, channel errors can alter the perception of sink of the outcome of the slot (due to interference and noise in the channel) in two major manners: a) The probability to hear a transmission when there is not one (false positive), is PeP; b) The probability to not hear an actual transmission (false negative) when a node sends a packet, is PeN.

\section{$5 \quad$ Simulation Results}

In this section some relevant numerical results are shown. Such results were obtained through a simulation based on discreet events according to each stage of the network life developed in $\mathrm{C}++$ language with the parameters described in section 4 . The main performance parameters considered in this section are the average cluster formation time and average energy consumption. Fig. 3a shows the average energy consumption in the system for 10 nodes inside the network and different probabilities of false positives and false negatives, both probabilities ranging from 0.01 to 0.1 .It can be 
seen that the false positive probability has a major impact on the system performance compared to the effect of the false negative probability. While variations of the false negative probability barely affects the energy consumption. A similar behavior is shown in Fig. $3 b$ which depicts the cluster formation time for the same 10 nodes network. This effect is not equal for both probabilities. Since false positive means a transmission, when none nodes transmit, the estimation of nodes attempting to transmit decreases causing high transmission probabilities for high number of nodes which entails more collisions on the system; causing a higher impact the false positive probability than the false negative probability. On the other hand, with the false negative probability, which means that a transmission is not detected, nodes retransmit the packets that were not correctly received by the sink with the optimal transmission probability because the estimation does not change.
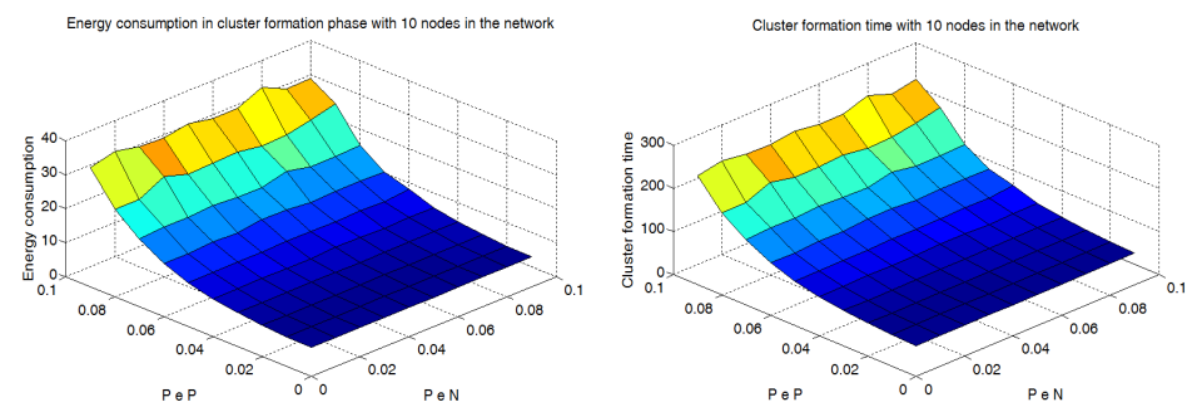

a)

b)

Fig. 3. a) Average cluster energy consumption under an adaptive probability of transmission with different channel errors, b) Average cluster formation time under an adaptive probability to transmission with different channel errors.

We also consider the case with 50 and 100 nodes in the WSN. When 50 nodes integrate the network, the affectation of both probabilities increases. Fig. 4a shows the average energy consumption for 50 nodes network with probabilities of false positive from 0.01 to 0.03 and probability of false negative from 0.01 to 0.1 . Also, Fig. 4b shows the average cluster formation time for the same network.

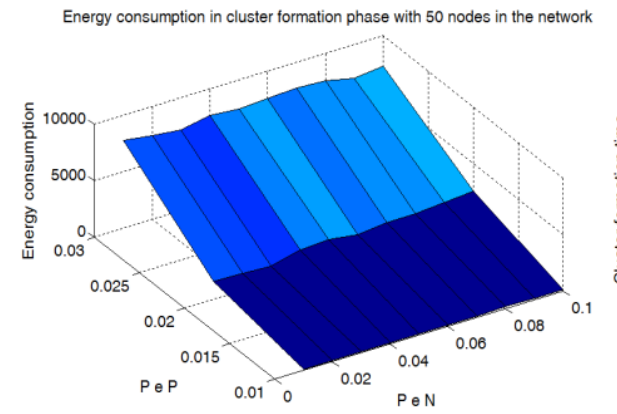

a)

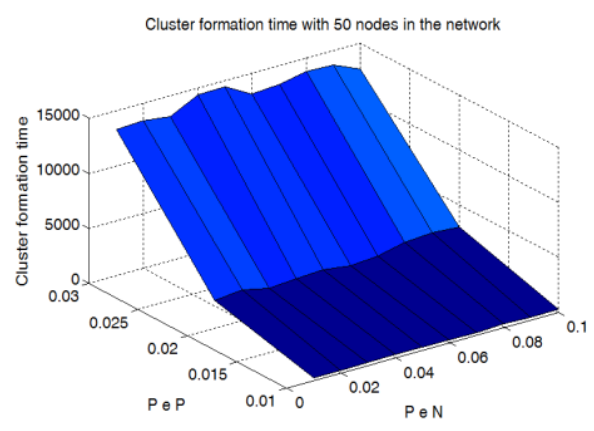

b)

Fig. 4. a) Average cluster energy consumption under an adaptive probability of transmission, b) Average cluster formation time under an adaptive probability of transmission. 
Note that for the scenario with 50 nodes in the network, the energy consumption is in the order of 10000 units when the false positive probability is 0.03 , while in the case where there are 10 nodes, the energy consumption is in the order of 10 units for the same value of false positive probability. The rationale behind these results is due to the density of the system. For instance, an error probability of 0.03 implies that for 100 transmissions, 3 of them will have error. Specifically, for the case of 10 nodes and assuming that all of them try to transmit, this probability of 0.03 represent that less than one transmission (0.3), will have error, but for the case of 50 nodes network, it implies that one or two transmissions (1.5) will have error. In such case, the rate of collisions increase more in the 50 nodes network since the sink detects more transmissions at the same time, even if only one node is transmitting. As the network density increases, the affectation of false positive and false negative probabilities too increases, as shown in Fig. 5a and in Fig. 5b. The following figures were obtained from a 100 nodes network with static probability of false positive of 0.01 and only changing the values of false negative probability, which ranges from 0.01 to 0.1.Unlike previous results, in the 100 nodes network, the affectation of false negative probability entails high rates of energy consumption and cluster formation time which are in the order of 10000 units.

Furthermore, the most error-free scenario where both probabilities are 0.01 has an energy consumption and cluster formation time of 5800 units and this increases to 13000 units. From these results it can be seen that the effect of the error estimation is important, especially for highly dense networks.

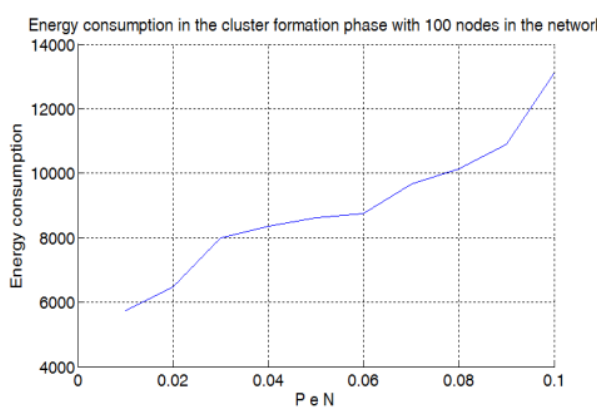

a)

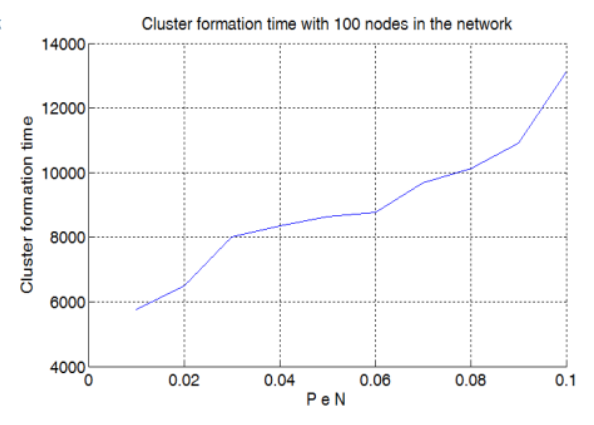

b)

Fig. 5. a) Average energy consumption under an adaptive probability of transmission, b) Average cluster formation time under an adaptive probability of transmission.

\section{Conclusions}

In this work, the LEACH-MOD protocol under an adaptive transmission probability with a noisy channel is studied. From the results obtained and presented, it is clear that a simple $\mathrm{CH}$ selection, like the LEACH-MOD protocol, can achieve a good system performance with energy consumption and complexity reductions even in the presence of errors in the wireless channel. Also, the use of the adaptive transmission strategy is of high importance to reduce energy consumption at the cluster formation phase. However, the presence of errors causes that energy consumption and cluster 
formation time increase, something that, inherently, impact the performance of our system.

Building on the obtained results, it is possible to see that errors in the channel affect more when the network increases in number of nodes. Furthermore, the effect of the false positive probability causes a higher impact than the false negative probability due to the fact that the false positives change the estimation of nodes attempting to transmit resulting in a high transmission probability when there is a high number of nodes remaining to transmit contrary to the false negative probability that does not change the estimation and nodes continue to transmit with the previously estimated probability.

\section{References}

1. Baronti, P., Pillai, P., Chook, V.W.C., Chessa, S., Gotta, A., Fun Hu, Y.: Wireless sensor networks: A survey on the state of the art and the 802.15.4 and ZigBee standards. Computer Communications, 30, 1655-1695 (2007)

2. Heinzelman, W.R., Chandrakasan, A., Balakrishnan, H.: Energy-efficient communication protocol for wireless microsensor networks. In: System Sciences, Proceedings of the 33rd Annual Hawaii International Conference, pp. 10 (2000)

3. Elhabyan, R.S., Yagoub, M.C.E.: Weighted tree based routing and clustering protocol for WSN. In: Electrical and Computer Engineering (CCECE), 26th Annual IEEE Canadian Conference, pp.1-6 (2013)

4. Nguyen, T.T., Shieh, C.S., Dao, T.K., Wu, J.S., Hu, W.C.: Prolonging of the Network Lifetime of WSN Using Fuzzy Clustering Topology. In: Robot, Vision and Signal Processing (RVSP), Second International Conference, pp. 13-16 ( 2013)

5. Romo-Montiel, E., Rivero-Ángeles, M.E., Villordo-Jiménez, I.: Effect of the error estimation of nodes in the cluster formation phase in wireless sensor networks with adaptive transmission probability. Research in Computing Science, 75, 9-18 (2014)

6. Handy, M.J., Haase, M., Timmermann, D.: Low energy adaptive clustering hierarchy with deterministic cluster-head selection. In: Mobile and Wireless Communications Network, 4th International Workshop, pp. 368-372 (2002)

7. Kredo, K., Mohapatra, P.: Medium access control in wireless sensor networks. Computer Networks, 50, 961-994 (2007)

8. Johnson, D.B., Maltz, D.A.: Truly seamless wireless and mobile host networking. Protocols for adaptive wireless and mobile networking. Personal Communications, IEEE, 3, 34-42 (1996)

9. Lin, R., Wang, Z., Sun, Y.: Energy efficient medium access control protocols for wireless sensor networks and its state-of-art. In: Industrial Electronics, IEEE International Symposium, pp. 669-674 (2004)

10. Lee H.C.: Towards a general wireless sensor network platform for outdoor environment monitoring. Sensors, IEEE, pp. 1-5 (2012) 